\title{
CORPOREIDAD Y GESTO EDUCATIVO: REPENSANDO LA ESCENA EN PERSPECTIVA SITUADA
}

Corporeality and educational gesture: Rethinking perspective scene located Corporalidade e gesto educacional: Cena Repensando perspectiva localizado

\section{Rodrigo Castillo Cuadra}

Universidad Católica del Norte, Chile. Fono: +56 2 209955. Correo electrónico: rcastillo@ucn.cl

\section{Resumen}

Sobre la dimensión de 'corporeidad' y 'gesto educativo' es que versará este artículo. Circularemos en torno al problema del extravío y olvido en que ha caído la noción de corporeidad para los sistemas educativos y para la cultura en general. La propuesta es delinear una lectura del fenómeno de la corporeidad como un ámbito simbólico significativo para la edificación de nuevas propuestas para la educación. Centrados en una reflexión educativa y filosófica daremos cuenta de cómo se construyen nuevos espacios de relación a partir de los entendimientos que aportan la fenomenología crítica, el arte y las ciencias cognitivas. Desde la cadena conceptual: Corporeidad, gesto y espacio educativo es que conduciremos este ensayo hacia una lectura situada sobre cómo la dimensión de corporeidad puede dar perspectivas para un nuevo escenario cultural.

Palabras Claves: Corporeidad, Gesto, Fenomenología, Situacionalidad.

\begin{abstract}
About the dimension of 'corporeality' and 'educational gesture' is that this article will talk about. We will circle around the problem of the loss and oversight in which the notion of corporeality for the educational system and culture in general has fallen off. The proposal is to draw up an interpretation of the phenomenom of corporeality as a significant symbolic aspect for the edification of new educational proposals. Based on an educational and philosophical eduaction we will show how new relationship spaces are constructed from the understandings that critical phenomenology, art and cognitive sciences provide. From the conceptual chain: 'Corporeality', 'gesture' and 'educational space' is where we will lead
\end{abstract}


this essay to an interpretation located on how the dimension of corporeality can provide different perspectives for a new cultural scene.

Key Words: Corporeality, Gesture, Phenomenology, Situationality.

\section{Resumo}

Sobre o tamanho de 'fisicalidade' e 'ato educativo' é que este artigo irá abordar. Circular em torno do problema perdido e esquecido que deixou cair à noção de corporalidade nos sistemas de ensino e cultura em geral. A proposta é delinear uma leitura do fenômeno da corporeidade como uma área simbólica significativa para a construção de novas propostas para a educação. Concentrando-se em uma reflexão educacional e filosófica vai perceber como novos espaços de relacionamento são construídos a partir dos entendimentos que fornecem a fenomenologia crítica, a arte e a ciência cognitiva. Desde o tripé conceitual: Personificação, gesto e espaço educacional que irá conduzir o ensaio definir uma leitura de como a dimensão física pode dar perspectivas de uma nova cena cultural.

Palavras-chave: Personificação, Gesto, Fenomenologia, situacionalidade.

\section{A modo de introducción: La Escena}

Nuestro encuadre es el panorama educativo actual en Chile; sobre él volcaremos nuestros trazos reflexivos convocados por las urgencias e importancias que tiene para nuestro país generar aportes que abran perspectivas para una educación que en su diagnóstico parece siempre agonizar. En mi caso la propuesta emerge desde el cuerpo, o más bien lo que denominaremos ‘corporeidad en vías de un gesto’ para la educación. Mi interés emana de las preguntas que la tradición educativa formal se ha ido realizando a lo largo de los años, pero más aún de las preguntas que ha olvidado; preguntas (las olvidadas) importantes para completar un camino reflexivo que instale ideas sólidas sobre las cuáles podamos repensar la educación. Nuevos repertorios, para nuevos procesos educativos. En este caso propongo un lineamiento que ha permanecido por años en la trastienda: a saber, la participación del cuerpo como dimensión fundante de procesos educativos en relación con procesos afectivos y motores que le permitan reintegrarse a una experiencia mucho mas vasta que llamaremos 'corporeidad', junto a todas sus implicancias afectivas que de su experenciar emanan. 
El escenario educativo está poblado de esfuerzos por parte de quienes piensan la escena por dar con fórmulas que nos garanticen buenos resultados en términos disciplinares. Me refiero a contenidos y dominios que muestren 'saberes' importantes de conseguir para insertarse en el mundo 'real'; propendemos el aumento de las capacidades y destrezas de nuestros estudiantes para operar en distintas realidades junto a una buena batería de actitudes que le permitan discernir y obrar de manera adecuada en el desarrollo de su plan vital. En esta línea constato la existencia de un tipo de procesos de 'enseñanza aprendizaje' centrado principalmente en las facultades que ejerce el dominio de la cognición entendida ésta como procesos de pensamiento orientados a la resolución de problemas lógico - matemáticos o lógico verbales los cuales no tienen ninguna vinculación con los planos corpóreos, sensibles y afectivos al interior del ser humano. La premisa desde donde arranca todo el proceso de 'enseñanza - aprendizaje' es orientar al estudiante al desarrollo de capacidades que le permitan sortear problemas estandarizados y previamente tipificados por el propio sistema educativo que luego, por medio de dispositivos de evaluación de 'conocimientos', son medidos para dar con la ponderación que corresponde. El montaje perfecto de una ficción en donde el sistema educativo confía que puede medir niveles de conocimientos de manera segmentada y separada, las que actúan con arreglo a resultados esperados. Todo completamente alejado del cómo funcionamos los seres humanos en espacios de relaciones, vinculares y emocionales. Al respecto se pronuncia desde las ciencias cognitivas a Francisco Varela, para deslindar los problemas que tendría el entender una cognición de esta manera:

El supuesto tácito de las diversas formas del realismo cognitivo (cognitivismo, emergencia, "sociedad de la mente") es que el mundo se puede dividir en regiones de elementos y tareas discretos. La cognición consiste en "resolver problemas", y esta resolución, para tener éxito, debe respetar los elementos, propiedades y relaciones de estas regiones pre - dadas (Varela et. al 1992: 174).

El citado autor pretende superar el inmovilismo en el que se encontraba la idea de cognición sostenida hasta entonces por toda la tradición del positivismo cognitivista. Varela avanza hacia el aporte de un concepto central para entender cómo el organismo opera en una red autónoma y contextualizada en donde la cognición se transforma en 'acción 
encarnada'; a saber: 'Enacción ${ }^{, 1}$. Un modo de entender la cognición en donde los patrones sensorio - motores más recurrentes se presentan en una modulación constante con la percepción y la acción del sujeto que conoce. La corporeidad sería un tejido de relaciones contextuales que operan no solo en dominios biológicos sino también relacionales generando un repertorio más amplio de procesos cognitivos.

En términos más específicos, el sistema nervioso está organizado mediante operaciones de cierre de una red de subredes modular y recíprocamente relacionadas, dando origen a conjunto de actividad coherentes tales como: i) patrones de correlación sensoriomotora que son estables, ii) una conducta para todo el organismo como una unidad móvil en el espacio. El cierre operacional del sistema nervioso produce un modo específico de coherencia, inscrustado en el organismo. Esta coherencia es un ser cognitivo: una unidad de percepción - movimiento en el espacio, de constantes senso- motoras medidas a través de la red de interneuronas. (...) La clave en este proceso cognitivo es el sistema nervioso a través de su neuro - lógica (Varela, 2002: 96).

Contrariamente vemos como el 'hecho educativo' tiene siempre como norte el modificar estructuras de pensamiento asumiendo un tipo de proceso cognitivo sin cuerpo, procesos carentes de movimiento y kinésica espacial; una kinésica que promueva el movimiento - encuentro y el movimiento - empatía. El 'hecho educativo' tiene como fundamento la necesidad de generar procesos de enseñanza - aprendizaje que desborden las técnicas de adiestramiento que, a lo único que apuntan es a generar tipos de respuestas psico-fisiológicas ante ciertos estímulos; sabemos que educar no es sólo crear mecanismos que midan procesos y que nos aseguren determinadas respuestas. No puede existir hecho educativo en lugares donde hayan procesos rutinarios que coharten los espacios de libertad y espontaneidad creativa del educando. El proceso educativo solo puede desarrollarse en un plano de libertad consciente y espiritual en los más vasto de la palabra, (racional y corpóreo), de ahí la gran responsabilidad de la educación en la tarea de preparar y disponer al ser humano para su propio encuentro, el encuentro con otros y con el mundo. El lugar simbólico donde se emplaza el hecho educativo tiene una geografía muy acotada; salas rectangulares, sillas y mesas dispuestas en orden geométrico, ordenadas en filas perpendiculares al pizarrón; todo apuntando hacia adelante, mirando el plano de una pizarra

${ }^{1}$ El neologismo "enacción” traduce el neologismo inglés enaction, derivado de enact, "representar", en el sentido de "desempeñar un papel, actuar." (Varela,1992:176) 
en donde se distingue la figura de un docente que se conduce horizontal frente a sus estudiantes vertiéndo sus puntos de vistas, diagnósticos e interpretaciones sobre una realidad dada, tomando para sí la idea de ser el guía y conductor de todo el proceso de aprendizaje. El supuesto desde donde nace esta idea, es que todos los (as) estudiantes se presentan como una suerte de 'tabla rasa' que deberá permanecer inmóvil, sentado, como receptáculo de todos los contenidos que el profesor debe entregar, negando toda posibilidad de movimiento e interacción como parte del proceso. Entonces la corporeidad y su repertorio gestual se dispone de manera vertical, tan vertical como un faro petrificado que ilumina en una sola dirección como una manera de disciplinar al cuerpo tal que pueda abrir paso a los procesos de pensamiento y sus constructos. Aquí el sistema escolar avala con fuerza la idea de que toda conducta a entrenar pasa por disciplinar al cuerpo en quietud, fijación y silencio. Repetida es la escena en donde el docente a punta de amenazas y castigos niega el movimiento de los estudiantes, los aquieta y acalla; en ese momento entiende que puede comenzar su clase. Fallamos en la mirada; promovemos una educación unidireccional, fija y pétrea; negamos lo mas vital que tiene todo proceso de pensamiento que es constituirse desde el movimiento y el encuentro. Una gran asamblea de procesos fisiológicos que tejen junto a las percepciones del mundo, este complejo proceso que llamamos conocer. Respecto de este punto, otro pronunciamiento desde las ciencias cognitivas que acompañamos en esta reflexión.

Así, se ha vuelto cada vez más necesario estudiar las neuronas como miembros de grandes conjuntos que constantemente desaparecen y surgen a través de interacciones cooperativas en donde cada neurona tiene múltilples y cambiantes grados de respuesta a los estímulos visuales, dependiendo del contexto. Aún en el extremo más periférico del sistema visual, las influencias que el cerebro recibe del ojo se encuentran con una mayor actividad que desciende del córtex. Es como resultado del encuentro de estos dos conjuntos de actividad neuronal que emerge una nueva y coherente configuración, dependiendo de la coincidencia o falta de coincidencia entre la actividad sensorial y el ambiente "interno" en el cortex. En general, una neurona individual participa en muchos de estos patrones globales y muestra una muy escasa significación cuando se la considera individualmente. (Varela, 2002: 194).

Entonces la pregunta es evidente: ¿por qué razón los procesos educativos se construyen a espaldas del movimiento y el cuerpo?; ¿cuál será la sospecha que impulsa a educar en un lugar fuera del 'conocimiento encarnado'?; finalmente y pecisando, ¿por qué 
no educar 'en y desde' el cuerpo y el movimiento? Sabemos que existe la obligatoriedad en los planes de estudio de dictar, 'Educación física y salud'; sin embargo estoy pensando en otro escenario, un currículo fundado a partir de una concepción de corporeidad que asuma por una parte el movimiento y por otra la dinámica corpórea como parte de la construcción de pensamiento. Un entronque entre los contenidos disciplinares y los espacios lúdicos y de interacción corpórea en donde se de un ensamble horizontal entre las prácticas pedagógicas tendientes a 'contenidos duros', y las prácticas o quehaceres en donde el cuerpo y el movimiento son protagonistas.

Postulo un lineamiento pedagógico que en su propuesta 'de puño y letra', señale mecanismos, didácticas y prácticas que permitan la recuperación de la experiencia educativa que surje de la síntesis entre las interacciones corporales y la integración de los contenidos disciplinares. Todo espacio pedagógico es un espacio de interacción. Esta recuperación de la experiencia pedagógica debiese constituirse en una narrativa corpórea que aporte una base de ideas sobre las cuáles comenzar a discutir y consensuar. Es el primer paso para la construcción de un acuerdo tácito acerca de cómo construir conocimiento. El lugar narrativo de la experiencia pedagógica permite que tanto cuerpo como lenguaje convivan en un espacio de relación en donde la actividad educativa es pretexto y la interacción entre educandos es texto. Se presentan entonces las condiciones para realizar una lectura simbólica en el eje sintagmático, (desenvolvimiento horizontal de la corporeidad); esto nos permite despejar cuál es el lugar en donde el cuerpo acontece como hecho simbólico en vías de la construcción de espacios de relaciones. La 'sincronía"2 que emerge a partir de la interacción corpórea necesariamente nos lleva a una experiencia horizontal en donde los estudiantes y todos los actores de los procesos educativos se disponen de manera extendida y abarcante para abordar todo el fenómeno que significa generar aprendizajes. Es importante entender los procesos educativos de manera 'horizontal' en su organización y despliegue , y no 'vertical' al modo como se lleva a cabo hoy amparado en jerarquías de todo tipo. Toda verticalidad en las relaciones humanas está

\footnotetext{
${ }^{2}$ El concepto de "sincronía" lo voy a entender desde el tratamiento fenomenológico que hace Emmanuel Lévinas a la idea de temporalidad, (Lévinas, 1993) con ocasión de reflexionar sobre la participación del existente en la realidad. En este sentido la temporalidad sincrónica resulta del fluir de las rememoranzas, las percepciones, los recuerdos que hacen presente los símbolos de la comunicación.
} 
dada para generar competitividad y no colaboración, los procesos humanos acontecen de manera sincrónica pues es de ese modo que vamos tejiendo signos en el decurso de la historia. Una definición para aclarar el punto: "La sincronía es el tiempo en el cual la conciencia, rememorando y presentificando las distintas fases temporales del fenómeno, reúne estas fases en el presente de la representación”. (Garrido - Maturano, 2000: 66).

\section{El “quehacer": Presente de la representación}

Lo que tenemos ahora es adoctrinamiento del cuerpo, gimnasia en sentido restringido; recordar que la noción de gimnasia proviene del vocablo griego 'gymnós', que significa desnudez; sabemos que para la tradición helena la desnudez era una expresión de la belleza, por tanto la 'gimnasia' refiere a una práctica física ejercida desde la desnudez del cuerpo todo conducido a alcanzar un componente espiritual que habitaría dentro del ser humano. Hoy las prácticas corpóreas están ligadas a la repetición de estímulos diseñados para dar cuenta de una orgánica estandarizada alejada de la construcción de espacios de descubrimiento de la experiencia corpórea en toda su maravilla y novedad. Hasta ahora 'educar el cuerpo' es abordarlo desde su tejido externo. Desde ese lugar se ha montado toda una programática centrada en mediciones sobre el rendimiento del cuerpo; es el cuerpo concebido como objeto estableciendo relaciones de competitividad; un tipo de cuerpo que actúa con arreglo a exhigencias externas que lo orientan al rendimiento; se trata de vencer al reloj, vencer la marca, la extensión, la presencia, el volúmen. No existe la concepción de un 'práctica' corpórea en el sentido de una praxis, como modo de obrar hacia un hacer transformador de la realidad desde lo corpóreo. La programática educativa a lo que propende es separar al cuerpo de los procesos de pensamiento, ningún profesor de asignaturas como por ejemplo, matemáticas, biología, lenguaje, pensaría fundar sus clases a partir de prácticas corpóreas. Nos sumimos en la idea de que cuerpo y despliegue es igual a recreación; y los paradigmas modernos en educación asocian recreación a distracción, pausa o descanso; momento para recuperar el aliento pues lo verdaderamente importante está por venir (pensar sentado mirando de frente al profesor). Llama la atención como la noción de 'recreo' en el ámbito escolar significa pausa entre momentos formales que son las lecciones de clases; el recreo es un momento asistemático, no organizado en donde el estudiante queda dispuesto para el descanso y la distracción. En general para los centros 
escolares el recreo es ruido, momento en que el cuerpo acontece como caos. "Existen diversas definiciones con respecto al concepto de 'recreo', las cuales coinciden con el hecho de que es un tiempo en el que los niños y niñas tienen una oportunidad para jugar y descansar del período formal de las clases”. (Cit. en Cháves Álvarez, 2013). Lo interesante es que lo estudiantes generalmente manifiestan interés por permanecer en esa pausa, extenderse y dar continuidad a un momento que 'se les pasa volando'. Me pregunto: ¿por qué no educar recreando?; ¿no es posible recuperar experiencia pedagógica instalando el recreo como lugar de convivencia dentro del aula? Aquí, nos encontramos con otra premisa equivocada y es la idea de que en pausa, la corporeidad cede junto a la cognición, por tanto no podríamos esperar aprendizaje en un espacio desorganizado. La experiencia que llevan adelante propuestas alternativas en educación que involucran las artes y los espacios lúdicos a los procesos educativos, están hablando de lo contrario; sólo en espacios de apertura y posibilitamiento de la exploración es que podemos fundar experiencia educativa y contrariamente esto no podría lograrse bajo espacios jerárquicos de control. Curioso, muy curioso pues señalamos antes que las estructuras de pensamiento funcionan totalmente distintos al orden planteado por el espacio educativo. Otro pronunciamiento para reforzar la crítica:

Un examen realizado mediante presencia plena/conciencia abierta revela que nuestra experiencia es discontínua: surge un momento de conciencia, parece permanecer un instante y se esfuma para ser reemplazado por el momento siguiente. ¿Esta descripción de la experiencia (la clase de descripción de la experiencia humana real que pedíamos) está en consonancia con las descripciones que nos brindan las neurociencias? Nótese que no hablamos de una dirección de causalidad. Y no recurrimos a las neurociencias para convalidar la experiencia, pues eso sería imperialismo científico. Simplemente nos interesa, de la manera más abierta posible, aquello que dicen las neurociencias acerca del problema de la momentaneidad (Varela et al. 1992: 98).

A mi juicio el error está en la base; obra a nivel de fundamento, desentenderse de la corporeidad y cómo esta dinamiza sus procesos como mente o conciencia en los procesos de aprendizaje es seguir afirmando un tipo de dualismo que hoy no se sostiene bajo ningún punto de vista. El ámbito educativo ha resultado ser el menos permeable frente a los cambios paradigmáticos que nacen de la discusión y los hallazgos al interior de la comunidad investigativa. Cuesta comprender como en pleno siglo XXI aún nos movamos bajo presupuestos tan antiguos en cuanto a considerar la importancia de la corporeidad y el 
vínculo en los espacios educativos. Constatamos acá el hecho de que la corporeidad se ha extraviado por siglos del panorama reflexivo por considerarla un simple estertor o colgajo de la razón, una materialidad de menor valía que solo contiene presencias mas importantes como la razón o el espíritu. Estos supuestos de los que parte toda la racionalidad moderna no son menores; son los ejes fundamentales de toda la arquitectura de pensamiento que tiene por cuerpo una idea sin importancia. En este sentido, me resulta importante mencionar al autor y la obra que a mi juicio pondrá el punto de inflexión más importante para revalorar la corporeidad en el universo reflexivo en Occidente. Me refiero a Maurice Merleau-Ponty y su texto "Phénoménologie de la perception" publicado en su primera versión en París en 1945. Sobre sus ideas es que versará el siguiente apartado.

\section{Revaloración de lo corpóreo: Reconstruyendo la escena ${ }^{3}$}

La propuesta en esta parte del texto es abrir otro ángulo para el problema en cuestión; sostuvimos una breve conversación con las neurociencias desde Francisco Varela; ahora me resulta necesario mirar el ámbito de la fenomenología crítica; en este sentido se instala de suyo relevante poner como soporte el primer intento sistemático que existe en la filosofía de pensar la corporeidad como un fenómeno integrado (relación sujeto - mundo perceptivo). El aporte lo hace Maurice Merleau-Ponty. De la lectura de sus obras más importantes me detendré en el análisis fenomenológico propuesto por él para tratar el concepto de 'cuerpo vivido' en unidad con el mundo perceptivo. Sabemos que el proyecto de pensamiento de Merleau - Ponty es de corte fenomenológico pero con una fuerte impronta existencial y crítica en su abordaje. Merleau - Ponty intenta establecer una filosofía que en tanto pensamiento nos lleve al descubrimiento de toda eventualidad que signifique observar el acontecer de la corporeidad en el mundo; es por eso que 'volver a las cosas mismas', resulta primordial pues es una invitación a volver a mirar o volver a encontrarse con aquello que acontece; una suerte de anterioridad experiencial en donde lo

\footnotetext{
3 Parte del presente apartado tienen su primer momento escritural en una tesis de Magistratura presentada bajo mi autoría al programa de "Magister Interdisciplinario en Estudios Latinoamericanos" de la Universidad de La Serena. En la ocasión tuve la oportunidad de llevar adelante un proyecto exploratorio en torno a la relación "corporeidad y gesto" inserto en escenarios culturales. El proyecto se encuentra ahora en etapa de revisión para su publicación.
} 
afirmado respecto de un estado de situación de la realidad no se interponga ni se superponga al encuentro entre sujeto cognoscente y mundo.

La fenomenología es el estudio de las esencias y, según ellas, todos los problemas se resuelven en la definición de esencias: la esencia de la percepción, la esencia de la conciencia, por ejemplo. Pero la fenomenología es asimismo una filosofía que re- sitúa las esencias dentro de la existencia y no cree que pueda comprenderse al hombre y al mundo más que a partir de su facticidad (Merleau-Ponty, 1975).

Lo interesante en Merleau-Ponty es que su proyecto asume un sentido de apertura de la conciencia hacia la existencia del hombre en el mundo. Hablamos de un intento concreto de pensar al ser humano en tanto condición corpórea inserto en la situacionalidad vital. Para ello nos invita a comprendernos más allá de los marcos explicativos que puede dar la ciencia acerca de mi cuerpo o de mi psiquismo. Debemos apelar directamente a la experiencia de cuerpo que tengo en el mundo para comenzar a trazar una idea de cómo los sujetos acontecemos en las relaciones. Ahora bien, es la percepción aquella fuente de apertura del sentido de mi corporeidad y su relación con el mundo, un tipo de percepción que me permite gestar una experiencia del habitar humano en el ámbito de una corporeidad vivida. La invitación en este sentido es a pensar al margen de una racionalidad depositada en un tipo de conciencia que piensa lo que somos y el cómo somos conciencia como una verdad anterior a lo 'ahí dado', que soy yo mismo viviéndome como cuerpo. Más bien se trata de comprendernos como una existencia desplegada en el mundo (realización de una existencia dada al mundo). Merleau - Ponty señala que como seres humanos somos la fuente absoluta desde donde nace toda comprensión; nuestra existencia no procede de los antecedentes que mi medio físico o social ha dado respecto de nosotros, más bien es nuestra existencia la que va hacia los antecedentes. Aquí tenemos el primer planteamiento crítico importante pues constato que aún heredamos fuertemente todo esta impronta cientificista de mediados del siglo XIX que influye e impacta el panorama reflexivo en educación que nos lleva a pensar desde las categorizaciones y demostraciones y no viceversa; ir desde la pregunta hacia las demostraciones. Es por esto que entro por la puerta que abre este autor como posibilidad de abordar esta pregunta por una corporeidad vivida; resulta importante poder 'suspender' el juicio para volver a mirar la corporeidad como un acontecimiento 
simbólico que revierte un carácter ético y comunicativo con toda la valía desde el pensar. Esta actitud originaria del pensamiento es la invitación a la que me sumo:

Volver a las cosas mismas es volver a este mundo antes del conocimiento del que el conocimiento habla siempre, y respecto del cual toda determinación científica es abstracta, significativa y dependiente, como la geografía respecto del paisaje en el que aprendimos por primera vez que era un bosque, un río o una pradera. (Merleau-Ponty, 1975).

La propuesta es reintegrar el cuerpo al universo perceptivo, no podemos seguir entendiendo al cuerpo como una parte separada de nuestra razón, o como un momento de la configuración del mundo psíquico; el cuerpo es un fenómeno integrado que es parte del tejido de nuestro entendimiento de todo cuanto existe. No nos preguntamos si percibimos de verdad un mundo; es decir no sospechamos que todo lo que se nos revela a nuestros sentidos pudiese ser una ilusión, por cierto distingo los sueños de la realidad pero todos estos elementos; más bien lo que puedo llamar 'mundo', es aquello que percibo en donde todas mis impresiones son correspondientes a las experiencias que de este mundo tengo. En este experienciar el mundo es donde el cuerpo se pliega al universo perceptivo pues es el cuerpo el lugar desde donde parte mi experiencia de mundo y no otro lugar. Decir experiencia de mundo es afirmar una experiencia de lo sensible (tacto, huella, piel, movimiento, agitación, memoria, órgano, percepción) en donde el cuerpo se manifiesta en una específicidad que le da espesor a mi sentir-aquejar el entorno.

Importantes obstáculos nos pone la cultura hoy para repensar la corporeidad en esta dirección. Me refiero a toda la carga estetizante y epidérmica que cae sobre el cuerpo como cánon estético o modelo de salud. Constato que en medio de la crisis moderna el cuerpo es depositario de todo tipo de lecturas que lo vacían; lecturas canónicas en términos de los dictámenes acerca de cómo debemos 'mantener' el cuerpo saludable. Si bien valoramos toda la información que nos ayuda a generar cuidados sobre el cuerpo, nuestro interés es montar una reflexión que supere las visiones puramente epidérmicas (lecturas del tejido), que terminan cosificando al cuerpo, ideas que dejan al cuerpo atrapado en un campo referencial que lo fija a fuego en un panorama interpretativo que lo extingue al punto de llegar a no ser considerado al momento de reflexionar sobre una reforma educativa, social o política. Este es el momento en que el cuerpo se transforma en sujeto - objeto, entra en un campo denotativo desde el cuál nada se puede decir y todo se puede padecer, el padecer del 
vacío, la enajenación, la alienación, un cuerpo sujeto venciendo al propio cuerpo como objeto. Bajo esta óptica ningún cambio estructural se podría pensar desde el cuerpo; vemos más bien un cuerpo victimizado y jalonado por un discurso publicitario que marca las expresiones del 'propio' cuerpo que hace imposible dar cuenta de una experiencia genuina y primaria respecto de como 'vivimos' la corporeidad; terminamos en un cuerpo despersonalizado, interpretado. Postulo un territorio en donde cuerpo y significaciones nos abran a un nuevo crisol de miradas renovadoras para la educación. Un tipo de corporeidad entendida como un sentido sensible y el gesto educativo como una declaración orgánica que presenta al cuerpo - pensamiento en toda su novedad. En tanto que 'cuerpo-sensible', está todavía del lado del sujeto que se percibe, se conoce y es invitado a aprehender desde el autoconocimiento y exploración de sí como un ser único; en tanto que 'cuerpo-sentido', está ya del lado de la cultura, en el escenario simbólico en donde se da toda la discusión acerca de cómo el cuerpo es depositario de todos los rastros de nuestra historia. Retornar al mundo perceptivo del cuerpo es comprender la relación primaria de la corporeidad con el mundo que es el 'sentido' y no el 'dominio'. El mundo perceptivo nos muestra que somos primariamente en el mundo en una relación de encuentro, apertura y develamiento tal que es, en ese encuentro y es, en esa apertura que el sentido del cuerpo inaugura su situación más próxima. Volcados desde ese lugar es que la percepción nos abre una ruta de comprensión de un sentido del cuerpo junto con sus posibilidades como libertad, como realización, como concreción. Merleau - Ponty en sus ensayos se aproxima de manera radical a la idea de que el mundo verdadero que se revela a la percepción corpórea no es el mundo de los estímulos lumínicos o las texturas y colores tal como los describe la ciencia; tampoco ese 'espectáculo de carne' ${ }^{4}$ que se da frente a nuestros ojos. Existe un mundo corpóreo más vasto en donde puedo vivenciar y comunicar volcado a la relación con otros, una experiencia de cuerpo colectivo fuera de las lecturas objetivantes. Dejamos de hablar de un 'cuerpo objetivo' para pasar a hablar de un 'cuerpo fenomenal'; un tipo de cuerpo que se mueve sin misterio en el mundo percibido pues es ya nuestro propio cuerpo experienciado como realidad vivida.

\footnotetext{
${ }^{4}$ Término empleado por Merleau-Ponty en un documento que forma parte de un conversatorio que es compendiado por Víctor Goldstein y reúne un set de siete conferencias que titula "El mundo percibido y el mundo de la ciencia”. (Merleau-Ponty, 2002).
} 
La palabra "aquí, aplicada a mi cuerpo, no designa una posición determinada con respecto a otras posiciones o con respecto a unas coordenadas exteriores, sino la instalación de las primeras coordenadas, el anclaje del cuerpo activo en un objeto, la situación del cuerpo ante sus tareas. El espacio corpóreo puede distinguirse del espacio exterior y envolver sus partes en lugar de desplegarlas porque este espacio es la oscuridad de la sala necesaria para la claridad del espectáculo, el fondo de somnolencia o la reserva de potencia vaga sobre los que se destacan el gesto y su objetivo, la zona de no - ser ante la cual pueden aparecer unos seres precisos, figuras y puntos (Merleau-Ponty, 1975:177).

Es en esta concepción de corporeidad donde se da la reunión de una subjetividad que percibe el mundo desde una intencionalidad que apunta a determinadas realidades; tenemos por ejemplo el acto de expresar, hablar desde el cuerpo y con el cuerpo acerca de como signamos el mundo, es esta expresión creación cultural en todo lo ancho de la palabra. Son, por nombrar como ejemplo; la poesía, la pintura, la danza, la música, el teatro, expresiones iluminadores de prácticas creativas en donde la corporeidad se rescata con la fuerza colectiva de la relación con otros. Son las artes en general las que de mejor manera han montado didácticas que favorecen dinámicas corpóreas dado que un trabajo de este tipo no se puede obtener en ausencia de un movimiento, un despliegue o una extensión que involucre todos los sentidos.

He tenido la oportunidad de conducir sistematicamente durante los último diez años, prácticas educativas volcadas al trabajo corpóreo al interior de un taller teatral en educación secundaria. Allí he podido observar lo que sucede cuando intencionadamente favoreces procesos de apertura de los sentidos en los estudiantes, que tengan que ver con abandonar el 'locus' de control del pensamiento en favor de una expresión corporal que permita la sincronía con procesos colectivos de creación. Estos procesos evidencian aprendizajes transversales complejos que no son medidos por los espacios formales en educación. ${ }^{5} \mathrm{Me}$ alojo fuera del espacio formal, sí al interior del centro escolar; actuando como un facilitador de procesos de creación colectiva cuidando siempre generar las mejores condiciones para permitir un desborde expresivo y corpóreo que nos permita visualizar recursos creativos sobre los cuáles poder volver para construir aprendizajes colectivos.

\footnotetext{
${ }^{5}$ Entiendo por espacios formales las "situaciones de aula", en donde el estudiante se centra netamente en el aprendizaje de contenido disciplinares y se da a la tarea de operacionalizar con esos conocimientos hacia objetivos esperados.
} 
Estos materiales se presentan como gestos, expresiones, relatos, testimonios, prácticas corpóreas, interacciones; en síntesis pensamiento en - acción. Interactuamos con objetos, dinamizamos el cuerpo en el espacio, construímos una poética en donde todos los trazos que el cuerpo dibuja en el espacio tengan un significado y un sentido, una coreografía del encuentro. Desde ese material experiencial comenzamos a tejer una interacción simbólica que es muy efectiva en tanto realidad que acontece para el grupo, pero que a su vez está poblada de significados nuevos, propios de una experiencia ficcional como lo es el teatro, favorecida por el juego y la impronta de representar de manera lúdica una realidad. Vamos poco a poco relevando a las palabras como enunciados verbalizados y junto a ello abandonamos lentamente la idea de que todo aprendizaje solo se puede expresar en el discurso. La palabra se pone al servicio de una acción corpórea que la antecede, como un subrayado sonoro de lo que el cuerpo 'dice' mediante los gestos. Sigo en esta línea una inspiración que viene desde la pedagogía teatral impulsada por Jacques Lecoq ${ }^{6}$

Abordamos las palabras por medio de los verbos, portadores de la acción, y por los nombres que representan a las cosas mencionadas. Considerando las palabras como un organismo vivo, buscamos el "cuerpo de las palabras". Para ello hay que escoger aquellas que ofrecen una verdadera dinámica corporal. Los verbos se prestan a esto más fácilmente: coger, levantar, romper, serrar son algunas de las muchas acciones que alimentan al verbo mismo. "Yo sierro" lleva en sí la dinámica del movimiento, En francés, le beurre (la mantequilla) ya está untada, mientras que en inglés, the butter está siempre en el envase. Según la lengua utilizada, las palabras no tienen la misma conexión con el cuerpo (Lecoq, 2009: 79).

Cada plano de este quehacer pedagógico que describo los vamos abordando paso a paso. Iniciamos en el descubrimiento del propio cuerpo y su memoria orgánica como huella de cómo el cuerpo deviene en la realidad particular de cada uno, para finalizar en la síntesis del contenido corporeizado en el espacio de relación. Darle un verdadero lugar al cuerpo en las artes de representación ha sido siempre complejo, en esta línea resuena la crítica que Antonin Artaud hacía a los procesos de construcción en el arte; situación que es análoga a la educación en el sentido de apostar todo a los contenidos y las palabras. Planteo que es

\footnotetext{
${ }^{6}$ Jacques Lecoq (París, 1921) estudia interpretación, mimo, expresión corporal y danza con el trabajo de Charles Dullin. En 1956 fundó en París su Escuela Internacional de Teatro, un centro de referencia de la pedagogía teatral del gesto y la palabra que inspiró a muchas escuelas de teatro en todo el mundo. Lecoq lleva adelante su proyecto de teatro corporal hasta su muerte en 1999.
} 
posible pensar la sala de clases, como lugar de representación escénica; también es posible pensar en cómo las ideas se escenifican y se corporeizan a nivel de los aprendizajes. Dejo la inquietud.

¿Cómo es posible que el teatro, tal como se le conoce en Europa, o mejor dicho en Occidente, haya menoscabado todo aquello que es propio de lo teatral, es decir, todo lo que no puede expresarse a través de palabras o, si se pretende, todo lo que no cabe en el diálogo y aun el diálogo mismo, como pasible de ser sonorizado expresivamente en escena y las "exigencias" de tal proceso? (Artaud, 2002: 37).

Retomando las ideas de este tipo de experiencia pedagógica, señalo que podemos observar cómo estos procesos de creación colectiva desde la corporeidad, son regulados de manera autónoma y aleatoria por los mismos estudiantes a medida que el cúmulo de experiencias va creciendo, todo en favor de un objetivo común que se traza al inicio de los talleres. Lo que vamos obteniendo capa tras capa son superficies de lenguajes que traen contenidos, otros traen relatos colectivos u otros solo movimientos y expresiones; luego comienza el ensamblaje de estas capas para visualizar la unidad de esta construcción. En todo momento la unidad es observada de manera colectiva y desde un lugar experiencial desde donde se prueba orgánicamente la fórmula y luego se conversa y discute. La fórmula es simple: comunicar un mensaje colectivo verdadero; instalar un verosímil ficcional que nos permita encontrarnos como grupo en un momento, y que luego permita a nuestros futuros espectadores encontrarse también. El actante que 'cuenta la historia' no es el personaje; apostamos por el colectivo narrativo, es el grupo el que cuenta la historia tal como sucede en el espacio comunitario; de esta manera el espacio ficcional se vuelve lo más cercano a cómo operan los sujetos en una cultura; no tenemos que hacer un esfuerzo por crear de espaldas a la realidad. Hablo de un relato orgánico, el todo más que las partes. Entonces y a propósito de lo expuesto, el esfuerzo va en la línea de entender y construir a partir de una subjetividad encarnada que promueva una fuerza creadora por un lado, y por otro asuma un protagonismo en la edificación del mundo. Hablo de una subjetividad que habita en un sujeto no desprevenido, un sujeto que se inscribe en un lugar relevante en la tarea de comenzar a 'decir' el mundo y este decir es corpóreo, una 'voz orgánica y encarnada'. 
La encarnación del pensar no es un accidente que le sucedería entorpeciéndole la tarea, al desviar de su lealtad el movimiento recto por el cual el pensar apunta al objeto. El cuerpo es el hecho que el pensar se sumerge en el mundo que piensa y, en consecuencia, expresa este mundo al mismo tiempo que lo piensa. El gesto corporal no es una descarga nerviosa, sino la celebración del mundo, poesía. El cuerpo es un sensible sentido - allí está, según Merleau - Ponty, su gran maravilla. En tanto que sentido, está todavía de este lado, del lado del sujeto; pero en tanto que sensible está ya de aquel lado, del lado los objetos; pensar que deja de ser paralítico, es movimiento que deja de ser ciego y comienza a ser creador de objetos culturales (Levinás, 2006: 31).

\section{Corporeidad y crítica: La encarnación de la escena}

Participar del mundo como corporeidad, es a la vez, leer el mundo y poder expresarlo por el gesto corpóreo anticipando al lenguaje por una especie de prolepsis $^{7}$, una anticipación del cuerpo y el gesto al lenguaje. Es la corporeidad la que acontece como experiencia fundante del conocimiento y no viceversa. Mientras tanto los espacios educativos siguen esperando que sea la razón la que gobierne todo el proceso educativo, nuestros estudiantes siguen experimentando ciegamente como se desentienden del lugar en donde todo proceso humano comienza: el cuerpo. La prolepsis; lo que se pueda expresar antes de las palabras no está considerado en la educación; todavía nos movemos en la dinámica del 'input - output'; cuidamos ciertas condiciones externas y esperamos por un resultado; mejor si el resultado es observable y medible. Otro error del que da cuenta Varela desde las neurociencias:

"En circunstancias muy restringidas es posible hablar como si pudiéramos especificar la operación de una célula u organismo a través de relaciones input/output. En general, sin embargo, el significado de esta o aquella interacción de un sistema viviente no está impuesto desde fuera, sino que es resultado de la organización e historia del sistema mismo" (Varela et al. 1992: 185).

La corporeidad como 'prolepsis' no responde a ninguno de estos circuitos, como inmediatez acontece anterior al circuito, se ubica como 'lateralidad'. Este movimiento permite la reconstrucción de nuevos significados del mundo de los cuáles la corporeidad participa en cuanto es capaz de desbordar al significado. Esta idea no es menor; antes de pensar en el predicado, la corporeidad como gesto acontece antes, en ese espacio líquido que precede al lenguaje. Por lo tanto y no siendo un enunciado sintagmático, el gesto

\footnotetext{
${ }^{7}$ En el sentido de acontecer anticipando la lectura referencial del enunciado, constituirse como un "decir" antes de la palabra.
} 
corpóreo entra en el lenguaje para darse a la tarea de levantar nuevos símbolos en un movimiento lateral. Siguiendo en este punto las ideas de Emannuel Levinás es que entiendo por un lado que ciertos órganos nos conectan con el mundo, pero por otra parte 'somos' todos esos órganos. Luego este acoplamiento orgánico nos da una espacialidad fundamental. Nos ubicamos en un punto desde donde nos percibimos tejiendo el mundo y la cultura. En ningún momento estamos desamblados, me refiero a ser órganos en un momento y luego participar del mundo; estamos totalmente integrados, es por esto que la ubicación del cuerpo en el espacio no lo agota; solo es un punto de referencia que le da una localización; luego volcado hacia el espacio simbólico el cuerpo aparece en distintos lugares y en distintos planos, esta es una de las cosas de la cuales Lévinas se maravillará: la “ubicuidad del cuerpo” (Lévinas, 2006) como la encarnación de los símbolos que hacen posible hablar del cuerpo como presencia.

La tarea es preguntarnos cómo podemos participar de la reconstrucción de nuevos significados para el mundo de la educación. Aquí la importancia del gesto corporal se presenta como ademán simbólico que cimenta la construcción de un espacio cultural y educativo lleno de significaciones. Es la propia corporeidad la que se va a poblar de significados nuevos en un ámbito en donde todas las experiencias sensibles conviven de manera aleatoria, sin orden de importancias; es decir, una experiencia plástica o kinésica tendrá tanto valor como una experiencia numérica y lógica para el proceso de aprendizaje. El estudiante aprende a disponerse desde el cuerpo para el aprendizaje, poco a poco va entiendiendo que no todas las operaciones lógico matemáticas o lógico verbales se dan en un discurso o en una operación abstracta sino también se concretan en una "cognición corporeizada" (Varela: 1992). Comenzamos a fundar un lugar reflexivo que rearme la categoría de 'hecho educativo' a partir del cuerpo. Esto nos pone en condiciónes de comprender que tanto corporeidad como movimiento, tanto lenguaje encarnado como emociones, le quitan petrificación a la estructura cognitiva que es apelada por la acción educativa a espera de resultados pedagógicos. Si comenzamos a incorporar la noción de movimiento estaremos aportando un dispositivo fundamental para una nueva forma de concebir el espacio educativo y la comunicación. Hasta ahora todas las expectativas están puestas en los contenidos. El plano sensible y visible de estos movimientos quedan relegados a un plano de menor importancia pues no apuntan necesariamente a verticalizar 
contenidos. En este punto es que apelo a la construcción de procesos de aprendizaje 'lateral', en donde el plano de la expresión como despliegue sea fundante de los contenidos; esta idea nos pone en condiciones de superar nuevos dualismos que están obrando sobre nuestros constructos. Dividir contenido y expresión como dos momentos distintos del cómo los sujetos aprendemos es una invitación a relevar un plano sobre otro en atención a la importancia. Error pues no existe tal preminencia, tanto contenido como expresión tienen una relación de equivalencia dado que no pueden cohexistir uno sin el otro. El problema es que desde la lectura cultural nos han enseñado a atender los contenidos y centrarnos en ello. Hoy día sabemos que los planos de expresión no son ya residuos fisiológicos de aquello que pasa a nivel 'interno'. La designación de un lugar 'interno' y otro 'externo' en el cuerpo se torna una ilustración vacía y sinsentido respecto de lo que el cuerpo realiza cuando comunica. Ideas importantes para ir desbancando el hecho de la 'representación' como reflejo de algo que supuestamente sucede dentro. Esta crítica tiene un desarrollo importante en la antropología simbólica de Ernst Cassirer; todo movimiento expresivo constituye un primer momento del desenvolvimiento espiritual en la medida en que se encuentra completamente situado en la inmediatez de la vida sensible y, por otra parte, va más allá de esta inmediatez (Cassirer, 1971). El movimiento expresivo implica un camino que en lugar de orientarse hacia un objetivo predeterminado sufre más bien una inhibición hacia un espacio distinto en el cual la corporeidad y el pensamiento pueden tomar conciencia de sí. Justamente me referí antes, a propósito de la práctica teatral, a la idea de que los 'aprendizajes esperados' frustran las posibilidades de expresión del plano corpóreo; los aprendizajes preconcebidos ceden frente a un tipo de objetivo colectivo y comunitario en donde el vivenciarse es parte del logro del proceso. En este sentido el cuerpo no se 'pos-pone' sino que se ubica como cuenco para generar la resonancia necesaria que alimenta el clima educativo.

La lectura del cuerpo en el ámbito educativo aún no tiene cabida, es un campo de significación abierta sin mayores referencias, por tanto toda incursión educativa en esta línea es valorable. Hasta ahora tenemos solo intentos furtivos y sabemos que 'una golondrina no hace verano'. Como lo señalé, partimos desde un cuerpo objetivado, lo entendemos como 'reflejo', un tipo de corporeidad que solo hace espejo del mundo; esta relación mimética es el sustento para generar toda la analogía entre pensamientos- 
contenidos y expresión-dispersión. Hoy hablamos desde otro lugar o más bien desde 'Otro' cuerpo. Una corporeidad que no va recogiendo rastrojos de un mundo dado y preconcebido; más bien un cuerpo que 'recusa' y se mueve en favor de un nuevo trazo que escribe una nueva historia. La historia del acontecer mágico (mágico en el sentido de producir movimientos extraordinarios que nos lleven a nuevas comprensiones de lo natural). La corporeidad como recusacion, significa que es el propio cuerpo vivido el que se desvincula de la tarea por construir aprendizajes verticales; el cuerpo se desambla, retrocede, no participa; a nivel de huella mnémica deja de incorporarse en un proyecto de aprendizaje pues siente a nivel fisiológico que el proyecto lo aliena. El proceso de alienación lo notamos a diario en las aulas; los estudiantes aprenden que no son capaces de hacer esto o aquello; 'aprehenden' a generar negaciones respecto de su acción pedagógica, incluso a veces no logran dar cuenta del por qué se sienten tan desvinculados o tan ajenos a los procesos educativos. También sitúo mi diálogo en esta dirección; que la tarea de reparación de todo proyecto educativo comience con una praxis transformadora de la realidad que tome carne y voluntad.

\section{Conclusiones: La bajada del telón}

Necesitamos abrir caminos en donde nuevas propuestas para la educación puedan convivir y dialogar; pasar de los diagnósticos al levantamiento de propuestas que teniendo su primer momento en lo ya andado, puedan en un segundo momento aportar ideas que generen miradas surtidoras de nutrientes para estos tiempos complejos. Mi propuesta va en la línea de la recuperación de una corporeidad vivida que sea significativa para la educación. Desde una mirada antropológica y simbólica podemos encontrar bases para construir una mirada integradora del cuerpo y el gesto en favor de la educación.

Es la dimensión simbólica del cuerpo el lugar donde debemos detenernos para repensar la escena de la educación; poder reflexionar sobre el valor del gesto corpóreo como una manifestación vívida generadora de sentidos al interior de la relación entre los seres humanos. Ésta valoración de la corporeidad como caudal comunicativo y de relación nos permitirá avanzar en la comprensión del fenómeno de acontecer como seres humanos en zonas más vastas que lo que captamos solo como conciencia. 
Plantear el gesto educativo como enunciado del cuerpo significa establecer una relación directa entre la corporalidad y la gestualidad como fenómenos integrados en la unidad de acontecer. La mirada que presento habla de esta unidad en donde el gesto se erije como acontecimiento fundamental en la experiencia de lo corpóreo. Inserto en la cultura, la dimensión simbólica del cuerpo se constituye como un espacio de recuperación de la humanidad de otros hombres en medio del decurso de la experiencia. Esta idea de recuperación del espacio de humanidad es la posibilidad de pensar un espacio educativo que tenga un tono ético y humano.

\section{Referencias Bibliográficas}

Artaud, A. (2003) El Teatro y su doble. México: Grupo Editorial Tomo.

Cassirer, E. (1971) Filosofía de las formas simbólicas Vol.1. México: Fondo de Cultura Económica.

Chaves, L. (2013). Una mirada a los recreos escolares: El sentir y pensar de los niños y niñas, Educare, 17, 1, 67-87.

Garrido-Maturano, A. (2000). Sincronía, diacronía y tiempo mesiánico. Génesis y evolución de la noción de tiempo en la Fenomenología de Emmanuel Lévinas. Revista Enfoques, 14, 1, 30-66.

Lecoq, J. (2009). El cuerpo poético. España: Alba Ed.

Lévinas, E. (1993). El tiempo y el otro, Barcelona: Paidós.

Levinás, E. (2006). Humanismo del otro Hombre. España: Siglo Veintiuno Editores.

Merleau - Ponty M. (1975). Fenomenología de la percepción. Barcelona: Península.

Merleau-Ponty M. (2002). El mundo de la percepción: Siete Conferencias. Buenos Aires: Fondo de Cultura Económica.

Varela, F. (2002). El fenómeno de la vida. Santiago de Chile: Dolmen.

Varela, F.; Thompson, E. y Rosch, E. (1992). De cuerpo presente. Barcelona: Gedisa. 